\title{
Rough Sets and Fuzzy Sets
}

\section{Preface}

This issue is dedicated to Professor Lotfi A. Zadeh, the founder of fuzzy set theory, on the occasion of celebrating 50 years of his first publication on fuzzy sets in 1965 [1]. The idea of fuzzy sets has influenced a very large number of researchers all over the world. Since 1965 many thousands of research papers on fuzzy set theory and its applications have been published as well as many thousands of patents based on fuzzy sets were issued (see http://www.cs.berkeley.edu/ zadeh/stimfl.html).

This special issue on Rough Sets and Fuzzy Sets presents some novel approaches and methodologies reflecting the state-of-the-art of rough sets, fuzzy sets, and their combination. After a rigorous review process out of 47 submissions from all over the world, 17 articles were selected for publication in this issue.

The rough set (RS) approach was proposed by Professor Zdzisław Pawlak in 1982 [2] as a tool for dealing with imperfect knowledge, in particular with vague concepts. Over the years many applications of methods based on RS theory alone or in combination with other approaches have been developed.

Fuzzy and rough set theories represent two different approaches to vagueness. Fuzzy set theory addresses a vague concept from the angle of gradualness of its nature, expressed by the fuzzy membership, whereas rough set theory addresses the same from the angle of its granular nature, expressed by the indiscernibility relation. In that sense both the theories are not competing each other, rather they are complementary to each other. Rough sets and fuzzy sets can work synergistically, often with other soft computing approaches.

Over the years many methods and applications, in particular in the field of pattern recognition, machine learning and data mining, were developed on the basis of rough sets, fuzzy sets and their combinations. The methods based on combination of the approaches exploit different abilities of mixed languages used for generating and expressing patterns by both the approaches. As the combination of these approaches provides better possibility of good approximation of the boundary region of a vague concept, it becomes possible to discover patterns of the higher quality in contrast to the situations when they are used in isolation. Rough-fuzzy (or fuzzy-rough) computing provides a stronger paradigm than 
fuzzy sets or rough sets, and can handle uncertainty arising both from the overlapping characteristics of concepts/classes/regions and granularity in the domain of discourse. The developed systems exploit the tolerance for imprecision, uncertainty, approximate reasoning and partial truth under soft computing framework and are capable of achieving tractability, robustness, and close resemblance with human like decision making for pattern recognition in ambiguous situations. The developed methods have found applications in many different domains. The objective of the rough-fuzzy integration is to provide a stronger paradigm for uncertainty handling in decision-making.

It is worthwhile mentioning that there is also a substantial progress in understanding the theoretical foundations of relationships between rough sets and fuzzy sets. The further research will certainly lead to the generation of more efficient methods based on combination of rough sets and fuzzy sets.

In order to help the readers for having better insight of this special issue, we provide brief overviews of the accepted papers. The papers can be classified into different groups with respect to different research topics.

The first group contains the research papers that concern the combination of both rough set theory and fuzzy set theory. Some of those papers focus on the theoretical properties and applications of the fuzzy rough set theory, i.e., generalizations of the classical rough set approach to fuzzy environments. The other papers either discuss on the hybridation of rough and fuzzy approaches to image processing or look for the common properties of both theories in the decision making process. This group consists of the following works:

- Structures of Opposition in Fuzzy Rough Sets

by Davide Ciucci, Didier Dubois and Henri Prade.

- Approximation-oriented Fuzzy Rough Set Approaches by Masahiro Inuiguchi;

- SoBT-RFW: Rough-Fuzzy Computing and Wavelet Analysis Based Automatic Brain Tumor Detection Method from MR Images

by Pradipta Maji and Shaswati Roy;

- Where Rough Sets and Fuzzy Sets Meet

by Lech Polkowski and Maria Semeniuk-Polkowska;

- Applications of Fuzzy Rough Set Theory in Machine Learning: a Survey by Sarah Vluymans, Yvan Saeys, Lynn Deer and Chris Cornelis;

- Using One Axiom to Characterize Fuzzy Rough Approximation Operators Determined by a Fuzzy Implication Operator by Wei-Zhi Wu, Tong-Jun Li and Shen-Ming Gu. 
The second group of papers concerns about the research in the main paradigms of rough set and fuzzy set theory, called approximation and information granulation. In particular, the authors investigate both theoretical properties and empirical aspects of lower and upper approximations as well as the fuzzy relations and information granulation. This topic is represented by the following papers:

- Generalized Quantifiers in the Context of Rough Set Semantics by Soma Dutta and Andrzej Skowron;

- On Quasi-Discrete Fuzzy Closure Spaces by Guilong Liu;

- Decision-theoretic Rough Sets-based Three-way Approximations of Interval-valued Fuzzy Sets by Guangming Lang, Tian Yang;

- Rough Set Approximations in Multi-granulation Fuzzy Approximation Spaces by Jian-Min Ma and Yiyu Yao;

- A Comparative Study of Multi-granulation Rough Sets (MGRSs) and Their Uncertainty Measures by Zhou-Ming Ma and Ju-Sheng Mi;

- Rough Approximations Based on Valued Tolerance Relations by Keyun Qin, Junfang Luo and Pei Zheng;

- T-Rough Approximation Pairs and Covering Based Rough Sets by Xiaohong Zhang, Yanning Zhang, Zhanao Xue and Yingcang Ma.

Information reduction under uncertainty is the common topic of the paper selected in the next group. These papers investigate the generalization of data reduction to the case of uncertain information systems. This part includes the following papers:

- Compression of dynamic fuzzy relation information systems by Mingjie Cai and Qingguo Li;

- Knowledge Reduction in Crisply Generated Fuzzy Concept Lattices by Mei-Zheng Li and Guoyin Wang;

- A Granular Computing Approach to Symbolic Value Partitioning by Liu-Ying Wen and Fan Min;

- Incremental computing approximations with the dynamic object set in interval-valued ordered information system by Jianhang Yu and Weihua Xu.

The editors of this issue are very grateful to Professor Lotfi A. Zadeh for his continuous support, in particular for his continuous stimulation in searching for complementary relationships of rough sets and fuzzy sets in order to make it possible to construct intelligent systems of better quality than those obtained by using each of the approaches separately. 


\section{References:}

[1] Zadeh, L.A.: Fuzzy sets. Information and Control 8, 338-353 (1965).

[2] Pawlak, Z.: Rough sets. International Journal of Computer and Information Sciences 11, 341-356 (1982)

\section{Editors of the issue}

\section{Chris Cornelis}

University of Granada

Dept. of Computer Science and AI

Centro de Investigación en Tecnologías de la

Información y las Comunicaciones (CITIC)

Email: Chris.Cornelis@ decsai.ugr.es

\section{Hung Son Nguyen}

Institute of Mathematics

University of Warsaw

Email: son@mimuw.edu.pl

\section{Sankar K. Pal}

Center for Soft Computing Research

Indian Statistical Institute, Kolkata

Email: sankar@isical.ac.in

Andrzej Skowron

Institute of Mathematics

University of Warsaw

Email: skowron@mimuw.edu.pl

\section{Wei-Zhi Wu}

School of Mathematics, Physics and Information Science Zhejiang Ocean University

Email:wuwz@zjou.edu.cn

October 2015 